\title{
The first passage time on the (reflected) Brownian motion with broken drift hitting a random boundary
}

\author{
Zhenwen Zhao ${ }^{\mathrm{a}}$, Yuejuan $\mathrm{Xi}^{\mathrm{a}, *}$ \\ ${ }^{a}$ School of Mathematical Sciences, Nankai University, Tianjin, PR China, 300071
}

\begin{abstract}
In this paper we consider a (reflected) Brownian motion with broken drift hitting a random boundary. Some dedicated calculations allow us to obtain the formula on the joint Laplace transform of the hitting time and hitting position. These develop the research on first rendezvous times of (reflected) Brownian motion and compound Poisson-type processes by Perry et al. (2004).
\end{abstract}

Keywords: Broken drift; the first passage time; Laplace transform

2010 MSC: 60J60, 60G40

\section{Introduction}

The reflected diffusion processes as a class of typical Markov processes have been widely applied in the subjects, such as queueing system, workload process, operation research and the others. Some references refer the reader to Harrison (1990), Jang \& Shim (2008), Perry et al. (2004), etc. On the other hand, the threshold process with piecewise drift term or diffusion term has also been another interesting class of processes (see, Su \& Chan (2015), Tong (1983), Yu et al. (2020)). Some specific threshold diffusion processes, for instance Brownian motion with alternative (broken) drift and Oscillating Brownian motion, were studied in Borodin \& Salminen (2002) and Keilson \& Wellner (1978).

The hitting boundary problem of a Markov process is a classical subject in the Markov process potential analysis. In recent years, most literature on the boundary crossing probability focuses on the linear or piecewise linear boundary (cf. Pötzelberger \& Wang (2001), Wang \& Pötzelberger (1997)). For a Markov process hitting a random boundary, Perry et al. (2004) formulated some results of the first rendezvous time of (reflected) Brownian motion (BM) and compound Poisson-type boundaries. Bo et al. (2011) extended the results in Perry et al. (2004) to the (reflected) Ornstein-Uhlenbeck process. Che \& Dassios (2013) discussed the problems of some boundary crossing probabilities for a Brownian motion with different stochastic boundaries, in particular, including compound Poisson process boundaries. In this paper, we consider a reflected Brownian motion (RBM) with broken drift hitting a random boundary. We will develop the above results in this direction.

Let us begin with a reflected diffusion process. Assume that $\left(\Omega, \mathcal{F}, \mathcal{F}_{t}, \mathcal{P}\right)$ is a probability space satisfying the usual conditions, and $W=\left\{W_{t}, t \geq 0\right\}$ is a standard Brownian motion. Define the diffusion

\footnotetext{
${ }^{*}$ Corresponding author

Email addresses: zzw@mail.nankai.edu.cn (Zhenwen Zhao), yjx@mail.nankai.edu.cn (Yuejuan Xi)
} 
processes $X=\left\{X_{t}, t \geq 0\right\}$ and $\tilde{X}=\left\{\tilde{X}_{t}, t \geq 0\right\}$ via the following stochastic differential equations (SDEs):

$$
\begin{aligned}
& d X_{t}=\mu\left(X_{t}\right) d t+d W_{t}, \\
& d \tilde{X}_{t}=\mu\left(\tilde{X}_{t}\right) d t+d W_{t}+d L_{t},
\end{aligned}
$$

where $\mu(x)=\mu_{1} \mathbf{1}_{\{x<c\}}+\mu_{2} \mathbf{1}_{\{x \geq c\}}, \mathbf{1}_{\{\cdot\}}$ is an indicator function, $\mu_{1}, \mu_{2} \in \mathbb{R}$ are constants and $c$ is a positve constant. The process $L=\left\{L_{t}: t \geq 0\right\}$ is the local time process of $X$ at the boundary zero and $L_{t}$ satisfies the property that

$$
L_{t}=\int_{0}^{t} \mathbf{1}_{\left\{\tilde{X}_{s}=0\right\}} d L_{s} \text { for all } t \geq 0,
$$

please see Ata et al. (2005), Harrison (1990) for details. We call $X$ and $\tilde{X}$ as Brownian motion with broken drift and reflected Brownian motion with broken drift respectively. The existence and uniqueness of the SDEs (1.1) and (1.2) were proved in Pilipenko (2014).

Next we introduce a random boundary $C(t)$ by

$$
C(t) \equiv b+Y \mathbf{1}_{\left\{T_{1} \leq t\right\}},
$$

where $b$ is a constant, $T_{1}$ admits an exponential law with parameter $\lambda>0$, which is independent of the Brownian motion $\mathrm{W}$. In addition, the random variable $Y$ is independent of $(T, W)$, which admits a distribution function given by $F(d y)$.

Define the hitting times:

$$
\begin{aligned}
& \tau=\inf \left\{t \geq 0: X_{t}=0 \text { or } X_{t}=C(t)\right\}, \\
& \tilde{\tau}=\inf \left\{t \geq 0: \tilde{X}_{t}=C(t)\right\},
\end{aligned}
$$

by convention, $\inf \varnothing=\infty$.

In Perry et al. (2004), they computed first rendezvous times in the case of the (reflected) BM. But for our case, due to the existence of the broken drift, using the conventional Meyer-Tanaka formula to construct martingale does not work. So we shall use time-dependent Meyer-Tanaka to derive first passage time of (reflected) BM hitting a random boundary in this paper.

The paper is organized as follows. In Section 2, we present and calculate some preliminary results of BM with broken drift and RBM with broken drift, respectively. In Section 3, we deal with the first passage time of a (reflected) Brownian motion with broken drift hitting a random boundary.

\section{Preliminaries}

In this section, we introduce some elementaries about the (reflected) BM with broken drift.

\subsection{The BM with broken drift}

Assume $X$ is the diffusion process defined in (1.1), then its infinitesimal generator defined on $C_{b}^{2}(\mathcal{R})$ should be

$$
\mathscr{A} f(x)=\frac{1}{2} f^{\prime \prime}(x)+\mu(x) f^{\prime}(x), \quad x \neq c .
$$


It follows from Borodin \& Salminen (2002, Page 128) that the speed measure of $X$ is

$$
m(d x)=\left(2 e^{2 \mu_{1} x} \mathbf{1}_{\{x<c\}}+2 e^{2\left(\mu_{1}-\mu_{2}\right) c} e^{2 \mu_{2} x} \mathbf{1}_{\{x \geq c\}}\right) d x
$$

and the scale density $s(x)$ is

$$
s(x)=\left(e^{-2 \mu_{1} x} \mathbf{1}_{\{x<c\}}+e^{2\left(\mu_{2}-\mu_{1}\right) c} e^{-2 \mu_{2} x} \mathbf{1}_{\{x \geq c\}}\right) d x .
$$

Define

$$
\psi_{s}(x)= \begin{cases}e^{\lambda_{1}^{+} x}, & x<c \\ A_{1} e^{\lambda_{2}^{-} x}+A_{2} e^{\lambda_{2}^{+} x}, & x \geq c\end{cases}
$$

and

$$
\varphi_{s}(x)= \begin{cases}B_{1} e^{\lambda_{1}^{-} x}+B_{2} e^{\lambda_{1}^{+} x}, & x<c, \\ e^{\lambda_{2}^{-} x}, & x \geq c,\end{cases}
$$

where the coefficients $\lambda_{1}^{+}, \lambda_{1}^{-}, \lambda_{2}^{+}, \lambda_{2}^{-}, A_{1}, A_{2}, B_{1}$ and $B_{2}$ are defined respectively by:

$$
\begin{array}{cc}
\lambda_{1}^{+}=-\mu_{1}+\sqrt{\mu_{1}^{2}+2 s}, & \lambda_{1}^{-}=-\mu_{1}-\sqrt{\mu_{1}^{2}+2 s}, \\
\lambda_{2}^{+}=-\mu_{2}+\sqrt{\mu_{2}^{2}+2 s}, & \lambda_{2}^{-}=-\mu_{2}-\sqrt{\mu_{2}^{2}+2 s} \\
A_{1}=\frac{\lambda_{2}^{+}-\lambda_{1}^{+}}{\lambda_{2}^{+}-\lambda_{2}^{-}} e^{\left(\lambda_{1}^{+}-\lambda_{2}^{-}\right) c}, & A_{2}=\frac{\lambda_{1}^{+}-\lambda_{2}^{-}}{\lambda_{2}^{+}-\lambda_{2}^{-}} e^{\left(\lambda_{1}^{+}-\lambda_{2}^{+}\right) c}, \\
B_{1}=\frac{\lambda_{1}^{+}-\lambda_{2}^{-}}{\lambda_{1}^{+}-\lambda_{1}^{-}} e^{\left(\lambda_{2}^{-}-\lambda_{1}^{-}\right) c}, & B_{2}=\frac{\lambda_{2}^{-}-\lambda_{1}^{-}}{\lambda_{1}^{+}-\lambda_{1}^{-}} e^{\left(\lambda_{2}^{-}-\lambda_{1}^{+}\right) c} .
\end{array}
$$

Then we can check $\psi_{s}($.$) and \varphi_{s}($.$) are the unique (up to a multiplicative constant) increasing and$ decreasing solutions of the following ordinary differential equation (ODE)

$$
\mathcal{A} f(x)=\frac{1}{2} f^{\prime \prime}(x)+\mu(x) f^{\prime}(x)=s f(x), x \neq c,
$$

which satisfy the boundary conditions $\mathcal{D}(\mathcal{A})=\left\{f: f, \mathcal{L} f \in \mathcal{C}_{b}(\mathcal{R}), f \in \mathcal{C}_{b}^{1}(\mathcal{R})\right\}$ (see the Theorem VII.3.12 in Revuz \& Yor (1999) for details).

Let $p(t, x, y)$ be the transition density function of $X$, and define $G_{s}(x, y)$ by

$$
G_{s}(x, y)=\int_{0}^{\infty} e^{-s t} p(t ; x, y) d t
$$

Then by Proposition 11 Borodin \& Salminen (2002, Section 2.1), $G_{s}(x, y)$ can be formulated as

$$
G_{s}(x, y)=\frac{m(y)}{\omega_{s}} \psi_{s}(x \wedge y) \varphi_{s}(x \vee y)
$$

where the Wronskian $\omega_{s}$ is a constant independent of $x$ and

$$
\omega_{s}=\frac{1}{s(x)}\left(\varphi_{s}(x) \psi_{s}^{\prime}(x)-\varphi_{s}^{\prime}(x) \psi_{s}(x)\right) .
$$

Next we can get the exact expression of the Green function $G_{s}(x, y)$. 
Proposition 2.1. (1) for $x<c$,

$$
G_{s}(x, y)= \begin{cases}\frac{2 e^{2 \mu_{1} y}}{\omega_{s}}\left(B_{1} e^{\lambda_{1}^{-} x}+B_{2} e^{\lambda_{1}^{+} x}\right) e^{\lambda_{1}^{+} y}, & y \leq x, \\ \frac{2 e^{2 \mu_{1} y}}{\omega_{s}}\left(B_{1} e^{\lambda_{1}^{-} y}+B_{2} e^{\lambda_{1}^{+} y}\right) e^{\lambda_{1}^{+} x}, & x<y<c \\ \frac{2 e^{2\left(\mu_{1}-\mu_{2}\right) c} e^{2 \mu_{2} y}}{\omega_{s}} e^{\lambda_{1}^{+}} x e^{\lambda_{2}^{-} y}, & y \geq c,\end{cases}
$$

(2) for $x \geq c$,

$$
G_{s}(x, y)= \begin{cases}\frac{2 e^{2 \mu_{1} y}}{\omega_{s}} e^{\lambda_{1}^{+} y} e^{\lambda_{2}^{-} x}, & y<c, \\ \frac{2 e^{2\left(\mu_{1}-\mu_{2}\right) c} e^{2 \mu_{2} y}}{\omega_{s}}\left(A_{1} e^{\lambda_{2}^{-} y}+A_{2} e^{\lambda_{2}^{+} y}\right) e^{\lambda_{2}^{-} x}, & c \leq y \leq x, \\ \frac{2 e^{2\left(\mu_{1}-\mu_{2}\right) c} e^{2 \mu_{2} y}}{\omega_{s}}\left(A_{1} e^{\lambda_{2}^{-} x}+A_{2} e^{\lambda_{2}^{+} x}\right) e^{\lambda_{2}^{-} y}, & y>x,\end{cases}
$$

where the Wronskian is

$$
\omega_{s}=\left(\lambda_{1}^{+}-\lambda_{2}^{-}\right) e^{\left(\lambda_{2}^{-}-\lambda_{1}^{-}\right) c} .
$$

Thus the transition density function $p(t ; x, y)$ of $X$ can follows from inverse Laplace transform as

$$
p(t ; x, y)=\mathcal{L}^{-1}\left(G_{s}(x, y)\right)(t) .
$$

This will be used in our derivations below.

\subsection{RBM with broken drift}

Recall $\tilde{X}$, a reflected Brownian motion $\tilde{X}$ with broken drift, defined in (1.2), and its infinitesimal generator for $f \in C_{b}^{2}(\mathcal{R})$ should be

$$
\mathscr{A} f(x)=\frac{1}{2} f^{\prime \prime}(x)+\mu(x) f^{\prime}(x), \quad x>0 \text { and } x \neq c .
$$

The speed measure of $\tilde{X}$ is

$$
m(d x)=\left(2 e^{2 \mu_{1} x} \mathbf{1}_{\{0 \leq x<c\}}+2 e^{2\left(\mu_{1}-\mu_{2}\right) c} e^{2 \mu_{2} x} \mathbf{1}_{\{x \geq c\}}\right) d x
$$

and the scale density $\tilde{s}(x)$ is

$$
\tilde{s}(x)=\left(e^{-2 \mu_{1} x} \mathbf{1}_{\{0<x<c\}}+e^{2\left(\mu_{2}-\mu_{1}\right) c} e^{-2 \mu_{2} x} \mathbf{1}_{\{x \geq c\}}\right) d x .
$$

Set

$$
\tilde{\psi}_{s}(x)= \begin{cases}\lambda_{1}^{+} e^{\lambda_{1}^{-} x}-\lambda_{1}^{-} e^{\lambda_{1}^{+} x}, & 0 \leq x<c \\ \tilde{A}_{1} e^{\lambda_{2}^{-} x}+\tilde{A}_{2} e^{\lambda_{2}^{+} x}, & x \geq c\end{cases}
$$

and

$$
\tilde{\varphi}_{s}(x)= \begin{cases}\tilde{B}_{1} e^{\lambda_{1}^{-} x}+\tilde{B}_{2} e^{\lambda_{1}^{+} x}, & 0 \leq x<c, \\ e^{\lambda_{2}^{-} x}, & x \geq c\end{cases}
$$

where the coefficients $\lambda_{1}^{+}, \lambda_{1}^{-}, \lambda_{2}^{+}, \lambda_{2}^{-}, \tilde{A}_{1}, \tilde{A}_{2}, \tilde{B}_{1}$ and $\tilde{B}_{2}$ are explicitly expressed by:

$$
\lambda_{1}^{+}=-\mu_{1}+\sqrt{\mu_{1}^{2}+2 s}, \quad \lambda_{1}^{-}=-\mu_{1}-\sqrt{\mu_{1}^{2}+2 s},
$$




$$
\begin{gathered}
\lambda_{2}^{+}=-\mu_{2}+\sqrt{\mu_{2}^{2}+2 s}, \quad \lambda_{2}^{-}=-\mu_{2}-\sqrt{\mu_{2}^{2}+2 s}, \\
\tilde{A}_{1}=\lambda_{1}^{+} \frac{\lambda_{2}^{+}-\lambda_{1}^{-}}{\lambda_{2}^{+}-\lambda_{2}^{-}} e^{\left(\lambda_{1}^{-}-\lambda_{2}^{-}\right) c}-\lambda_{1}^{-} \frac{\lambda_{2}^{+}-\lambda_{1}^{+}}{\lambda_{2}^{+}-\lambda_{2}^{-}} e^{\left(\lambda_{1}^{+}-\lambda_{2}^{-}\right) c}, \\
\tilde{A}_{2}=\lambda_{1}^{+} \frac{\lambda_{1}^{-}-\lambda_{2}^{-}}{\lambda_{2}^{+}-\lambda_{2}^{-}} e^{\left(\lambda_{1}^{-}-\lambda_{2}^{+}\right) c}-\lambda_{1}^{-} \frac{\lambda_{1}^{+}-\lambda_{2}^{-}}{\lambda_{2}^{+}-\lambda_{2}^{-}} e^{\left(\lambda_{1}^{+}-\lambda_{2}^{+}\right) c}, \\
\tilde{B}_{1}=\frac{\lambda_{1}^{+}-\lambda_{2}^{-}}{\lambda_{1}^{+}-\lambda_{1}^{-}} e^{\left(\lambda_{2}^{-}-\lambda_{1}^{-}\right) c}, \quad \tilde{B}_{2}=\frac{\lambda_{2}^{-}-\lambda_{1}^{-}}{\lambda_{1}^{+}-\lambda_{1}^{-}} e^{\left(\lambda_{2}^{-}-\lambda_{1}^{+}\right) c} .
\end{gathered}
$$

Then we can check $\tilde{\psi}_{s}($.$) and \tilde{\varphi}_{s}($.$) are the unique (up to a multiplicative constant) increasing and$ decreasing solutions of the following ordinary differential equation (ODE)

$$
\mathcal{L} f(x)=\frac{1}{2} f^{\prime \prime}(x)+\mu(x) f^{\prime}(x)=s f(x), x>0 \text { and } x \neq c,
$$

which satisfy the boundary conditions $\mathcal{D}(\mathcal{L})=\left\{f: f, \mathcal{L} f \in \mathcal{C}_{b}([0,+\infty)), f^{\prime}(0+)=0, f^{\prime}(c-)=f^{\prime}(c+)\right\}$.

Similarly, from (2.2) and (2.3) we can get the Green function $\tilde{G}_{s}(x, y)$.

Proposition 2.2. (1) for $0<x<c$,

$$
\tilde{G}_{s}(x, y)= \begin{cases}\frac{2 e^{2 \mu_{1} y}}{\tilde{\omega}_{s}}\left(\tilde{B}_{1} e^{\lambda_{1}^{-} x}+\tilde{B}_{2} e^{\lambda_{1}^{+} x}\right)\left(\lambda_{1}^{+} e^{\lambda_{1}^{-} y}-\lambda_{1}^{-} e^{\lambda_{1}^{+} y}\right), & 0<y \leq x, \\ \frac{2 e^{2 \mu_{1} y}}{\tilde{\omega}_{s}}\left(\tilde{B}_{1} e^{\lambda_{1}^{-} y}+\tilde{B}_{2} e^{\lambda_{1}^{+} y}\right)\left(\lambda_{1}^{+} e^{\lambda_{1}^{-} x}-\lambda_{1}^{-} e^{\lambda_{1}^{+} x}\right), & x<y<c, \\ \frac{2 e^{2\left(\mu_{1}-\mu_{2}\right) c} e^{2 \mu_{2} y}}{\tilde{\omega}_{s}}\left(\lambda_{1}^{+} e^{\lambda_{1}^{-} x}-\lambda_{1}^{-} e^{\lambda_{1}^{+} x}\right) e^{\lambda_{2}^{-} y}, & y \geq c,\end{cases}
$$

(2) for $x \geq c$,

$$
\tilde{G}_{s}(x, y)= \begin{cases}\frac{2 e^{2 \mu_{1} y}}{\tilde{\omega}_{s}}\left(\lambda_{1}^{+} e^{\lambda_{1}^{-} y}-\lambda_{1}^{-} e^{\lambda_{1}^{+} y}\right) e^{\lambda_{2}^{-} x}, & 0<y<c, \\ \frac{2 e^{2\left(\mu_{1}-\mu_{2}\right) c} e^{2 \mu_{2} y}}{\tilde{\omega}_{s}}\left(\tilde{A}_{1} e^{\lambda_{2}^{-} y}+\tilde{A}_{2} e^{\lambda_{2}^{+} y}\right) e^{\lambda_{2}^{-} x}, & c \leq y \leq x, \\ \frac{2 e^{2\left(\mu_{1}-\mu_{2}\right) c} e^{2 \mu_{2} y}}{\tilde{\omega}_{s}}\left(\tilde{A}_{1} e^{\lambda_{2}^{-} x}+\tilde{A}_{2} e^{\lambda_{2}^{+} x}\right) e^{\lambda_{2}^{-} y}, & y>x\end{cases}
$$

where the Wronskian is

$$
\tilde{\omega}_{s}=-\lambda_{1}^{+}\left(\lambda_{2}^{-}-\lambda_{1}^{-}\right) e^{\left(\lambda_{2}^{-}-\lambda_{1}^{+}\right) c}-\lambda_{1}^{-}\left(\lambda_{1}^{+}-\lambda_{2}^{-}\right) e^{\left(\lambda_{2}^{-}-\lambda_{1}^{-}\right) c} .
$$

Thus the transition density function $\tilde{p}(t ; x, y)$ of $\tilde{X}$ is denoted by

$$
\tilde{p}(t ; x, y)=\mathcal{L}^{-1}\left(\tilde{G}_{s}(x, y)\right)(t) .
$$

\section{Hitting problems of BM with broken drift and RBM with broken drift}

\subsection{Hitting problem of BM with broken drift}

In this subsection, we shall study the first passage time $\tau$ defined in (1.4). As in Perry et al. (2004), we will compute the joint Laplace transform of the random vector $\left(X_{\tau}, \tau\right)$, i.e.,

$$
\Psi(\alpha, \theta ; x)=\mathbf{E}_{x}\left[e^{-\alpha X_{\tau}-\theta \tau}\right]
$$


where $\mathbf{E}_{x}[\cdot]=\mathbf{E}\left[\cdot \mid X_{0}=x\right]$. For this purpose, we are first to compute the following four Laplace transforms:

$$
\begin{aligned}
\Phi_{1}(\alpha, \theta ; x) & =\mathbf{E}_{x}\left[e^{-\alpha X_{T_{1}}-\theta T_{1}} \mathbf{1}_{\left\{X_{\left.T_{1}<c\right\}}\right\}}\right], \\
\Phi_{2}(\alpha, \theta ; x) & =\mathbf{E}_{x}\left[e^{-\alpha X_{T_{1}}-\theta T_{1}} \mathbf{1}_{\left\{X_{T_{1} \geq c}\right\}}\right], \\
\Phi_{3}(\alpha, \theta ; x) & =\mathbf{E}_{x}\left[e^{-\alpha X_{T_{1}}-\theta T_{1}} \mathbf{1}_{\left\{X_{\left.T_{1}<c\right\}}\right\}} \mathbf{1}_{\left\{\tau<T_{1}\right\}}\right], \\
\Phi_{4}(\alpha, \theta ; x) & =\mathbf{E}_{x}\left[e^{-\alpha X_{T_{1}}-\theta T_{1}} \mathbf{1}_{\left\{X_{T_{1} \geq c}\right\}} \mathbf{1}_{\left\{\tau<T_{1}\right\}}\right] .
\end{aligned}
$$

On the other hand, we need these two identities:

$$
\begin{aligned}
& \omega_{1}(\theta ; b, x)=\mathbf{E}_{x}\left[e^{-\theta R_{0}} \mathbf{1}_{\left\{R_{0}<R_{b}\right\}}\right], \\
& \omega_{2}(\theta ; b, x)=\mathbf{E}_{x}\left[e^{-\theta R_{b}} \mathbf{1}_{\left\{R_{0}>R_{b}\right\}}\right]
\end{aligned}
$$

where $R_{a}$ denotes the first passage time of the stochastic process $X=\left\{X_{t}: t \geq 0\right\}$ over a constant boundary, i.e., $R_{a}=\inf \left\{t \geq 0: X_{t}=a\right\}$ for $a \in \mathcal{R}^{1}$.

If $0<x<b$, since the function $\psi_{\theta}(x)$ and $\varphi_{\theta}(x)$ are the solutions to the ordinary differential equation (ODE) (2.1), applying Itô's formula and the optional stopping theorem yields:

$$
\begin{aligned}
\mathbf{E}_{x}\left[e^{-\theta\left(R_{0} \wedge R_{b}\right)} \psi_{\theta}\left(X_{R_{0} \wedge R_{b}}\right)\right] & =\psi_{\theta}(x), \\
\mathbf{E}_{x}\left[e^{-\theta\left(R_{0} \wedge R_{b}\right)} \varphi_{\theta}\left(X_{R_{0} \wedge R_{b}}\right)\right] & =\varphi_{\theta}(x) .
\end{aligned}
$$

So we can obtain

$$
\begin{aligned}
& \psi_{\theta}(x)=\psi_{\theta}(0) \omega_{1}(\theta ; b, x)+\psi_{\theta}(b) \omega_{2}(\theta ; b, x), \\
& \varphi_{\theta}(x)=\varphi_{\theta}(0) \omega_{1}(\theta ; b, x)+\varphi_{\theta}(b) \omega_{2}(\theta ; b, x) .
\end{aligned}
$$

Solving $\omega_{1}(\theta ; b, x), \omega_{2}(\theta ; b, x)$ in $(3.3)$ and (3.4), we yield the expression of $\omega_{1}(\theta ; b, x), \omega_{2}(\theta ; b, x)$ :

$$
\omega_{1}(\theta ; b, x)=\frac{\varphi_{\theta}(x) \psi_{\theta}(b)-\psi_{\theta}(x) \varphi_{\theta}(b)}{\varphi_{\theta}(0) \psi_{\theta}(b)-\psi_{\theta}(0) \varphi_{\theta}(b)}
$$

and

$$
\omega_{2}(\theta ; b, x)=\frac{\varphi_{\theta}(x) \psi_{\theta}(0)-\psi_{\theta}(x) \varphi_{\theta}(0)}{\varphi_{\theta}(b) \psi_{\theta}(0)-\psi_{\theta}(b) \varphi_{\theta}(0)} .
$$

We introduce the following time-dependent Meyer-Tanaka formula derived in Elworthy et al. (2007).

Lemma 3.1. If $f(t, x)$ satisfies the following conditions.

(1) $f$ is absolutely continuous in each variable.

(2) $\partial_{t}^{-} f$ and $\partial_{x}^{-} f$ exist, are left-continuous and locally bounded.

(3) $\partial_{x}^{-} f$ is of locally bounded variation in $\mathbb{R}_{+} \times \mathbb{R}$ and $\partial_{x}^{-} f(0, \cdot)$ is of locally bounded variation in $\mathbb{R}$.

Then we have the following extension meyer-tanaka formula to time-dependent functions:

$$
\begin{aligned}
f\left(t, X_{t}\right) & =f\left(0, X_{0}\right)+\int_{0}^{t} \partial_{t}^{-} f\left(s, X_{s}\right) d s+\int_{0}^{t} \partial_{x}^{-} f\left(s, X_{s}\right) d X_{s} \\
& +\int_{\mathbb{R}} L^{X}(t, y) d_{y} \partial_{x}^{-} f(t, y)-\int_{\mathbb{R}} \int_{0}^{t} L^{X}(s, y) d_{s, y} \partial_{x}^{-} f(s, y),
\end{aligned}
$$


where the local time is defined by the limit in probability:

$$
L^{X}(t, y)=\frac{1}{2} \int_{0}^{t} \delta\left(X_{s}-y\right) d\langle X\rangle_{s}
$$

where $\delta(x)=0,(x \neq 0)$ and $\int_{-\infty}^{\infty} \delta(x) d x=1$. The notations $d_{y}$ and $d_{s, y}$ mean integration with respect to the $y$ variable and the $(s, y)$ variables, respectively.

According to the Theorem 2.1 of Bo et al. (2011) and Theorem 3.1 of Perry et al. (2004), we have the following result.

Theorem 3.1. Let $0<x<b$. Then the joint LT of $\left(X_{\tau}, \tau\right)$ is given by

$$
\Psi(\alpha, \theta ; x)=\omega_{1}(\lambda+\theta ; b, x)+e^{-\alpha b} \omega_{2}(\lambda+\theta ; b, x)+\mathbf{E}_{x}\left[e^{-\theta T_{1}} M\left(\theta, X_{T_{1}}, Y_{1}\right) \mathbf{1}_{\left\{\tau \geq T_{1}\right\}}\right],
$$

where the function $M$ is defined by

$$
M(\theta, x, y)=\omega_{1}(\lambda+\theta ; b+y, x)+e^{-\alpha(b+y)} \omega_{2}(\lambda+\theta ; b+y, x), y>0
$$

the functions $\omega_{1}$ and $\omega_{2}$ are defined in (3.5) and (3.6). The last term on the right side of (3.8) can be determined by the following Proposition 3.1 and Proposition 3.2.

Next, we are ready to prove the Theorem 3.1. By using the Lemma 3.1, we can obtain the LTs evaluated at the exponential random time $T_{1}$, i.e., $\Phi_{1}(\alpha, \theta ; x)$ and $\Phi_{2}(\alpha, \theta ; x)$.

Proposition 3.1. Let $x \in \mathbb{R}$ and $\alpha, \theta>0$. Then

$$
\begin{aligned}
& \Phi_{1}(\alpha, \theta ; x)=\frac{\lambda\left(e^{-\alpha x} \mathbf{1}_{\{x<c\}}+e^{-\alpha c} \mathbf{1}_{\{x \geq c\}}\right)-(\lambda+\theta) e^{-\alpha c} g(\theta ; x)}{\lambda+\theta+\alpha \mu_{1}-\frac{1}{2} \alpha^{2}}, \\
& \Phi_{2}(\alpha, \theta ; x)=\frac{\lambda\left(e^{-\alpha x} \mathbf{1}_{\{x \geq c\}}-e^{-\alpha c} \mathbf{1}_{\{x \geq c\}}\right)+(\lambda+\theta) e^{-\alpha c} g(\theta ; x)}{\lambda+\theta+\alpha \mu_{2}-\frac{1}{2} \alpha^{2}},
\end{aligned}
$$

where

$$
g(\theta ; x)=\mathbf{E}_{x}\left[e^{-\theta T_{1}} \int_{c}^{\infty} p\left(T_{1} ; x, y\right) d y\right]
$$

and $p(t ; x, y)$ is the transition density function for the BM with broken drift (i.e. stochastic process $X$ ) that can be obtained in (2.4).

Proof. By using Itô's formula to $e^{-\alpha X_{t}-\theta t}$, we have

$$
M_{t}=e^{-\alpha X_{t}-\theta t}-e^{-\alpha X_{0}}+\left(\theta-\frac{1}{2} \alpha^{2}\right) \int_{0}^{t} e^{-\alpha X_{s}-\theta s} d s+\alpha \int_{0}^{t} \mu\left(X_{s}\right) e^{-\alpha X_{s}-\theta s} d s
$$

is a martingale. Now, applying the optional stopping time theorem to $M$ and $T_{1}$, we obtain

$$
\Phi_{1}(\alpha, \theta ; x)+\Phi_{2}(\alpha, \theta ; x)-e^{-\alpha x}+\left(\theta-\frac{1}{2} \alpha^{2}\right) \mathbf{E}_{x}\left[\int_{0}^{T_{1}} e^{-\alpha X_{s}-\theta s} d s\right]+\alpha \mathbf{E}_{x}\left[\int_{0}^{T_{1}} \mu\left(X_{s}\right) e^{-\alpha X_{s}-\theta s} d s\right]=0 .
$$

Since the exponential random variable $T_{1}$ is independent of the process $X$, we have

$$
\mathbf{E}_{x}\left[\int_{0}^{T_{1}} e^{-\alpha X_{s}-\theta s} d s\right]=\frac{1}{\lambda}\left(\Phi_{1}(\alpha, \theta ; x)+\Phi_{2}(\alpha, \theta ; x)\right)
$$


and

$$
\mathbf{E}_{x}\left[\int_{0}^{T_{1}} \mu\left(X_{s}\right) e^{-\alpha X_{s}-\theta s} d s\right]=\frac{1}{\lambda} \mathbf{E}_{x}\left[\mu\left(X_{T_{1}}\right) e^{-\alpha X_{T_{1}}-\theta T_{1}}\right] .
$$

Thus, we yield the following equation

$$
\frac{\lambda+\theta+\alpha \mu_{1}-\frac{1}{2} \alpha^{2}}{\lambda} \Phi_{1}(\alpha, \theta ; x)=e^{-\alpha x}-\frac{\lambda+\theta+\alpha \mu_{2}-\frac{1}{2} \alpha^{2}}{\lambda} \Phi_{2}(\alpha, \theta ; x) .
$$

Define the function $h(t, x)$ by $h(t, x)=e^{-\alpha x-\theta t} \mathbf{1}_{\{x<c\}}+e^{-\alpha c-\theta t} \mathbf{1}_{\{x \geq c\}}$. Obviously, $h(t, x)$ satisfies the conditions in Lemma 3.1, as a consequence, we yield that

$$
\begin{aligned}
N_{t} & =e^{-\alpha X_{t}-\theta t} \mathbf{1}_{\left\{X_{t}<c\right\}}+e^{-\alpha c-\theta t} \mathbf{1}_{\left\{X_{t} \geq c\right\}}-e^{-\alpha x} \mathbf{1}_{\{x<c\}}-e^{-\alpha c} \mathbf{1}_{\{x \geq c\}} \\
& +\left(\alpha \mu_{1}+\theta\right) \int_{0}^{t} e^{-\alpha X_{s}-\theta s} \mathbf{1}_{\left\{X_{s} \leq c\right\}} d s+\theta \int_{0}^{t} e^{-\alpha c-\theta s} \mathbf{1}_{\left\{X_{s}>c\right\}} d s- \\
& \alpha^{2} \int_{\mathbb{R}} L^{X}(t, y) e^{-\alpha y-\theta t} \mathbf{1}_{\{y \leq c\}} d y-\alpha^{2} \theta \int_{\mathbb{R}} \int_{0}^{t} L^{X}(s, y) e^{-\alpha y-\theta s} \mathbf{1}_{\{y \leq c\}} d s d y,
\end{aligned}
$$

is a martingale. By using the occupation times formula, we obtain

$$
\int_{\mathbb{R}} L^{X}(t, y) e^{-\alpha y-\theta t} \mathbf{1}_{\{y \leq c\}} d y=\frac{1}{2} e^{-\theta t} \int_{0}^{t} e^{-\alpha X_{s}} \mathbf{1}_{\left\{X_{s} \leq c\right\}} d s
$$

and similarly

$$
\int_{\mathbb{R}} \int_{0}^{t} L^{X}(s, y) e^{-\alpha y-\theta s} \mathbf{1}_{(y \leq c)} d s d y=\frac{1}{2} \int_{0}^{t} \int_{0}^{s} e^{-\alpha X_{u}} \mathbf{1}_{\left\{X_{u} \leq c\right\}} d u e^{-\theta s} d s .
$$

Then, applying the optional stopping time theorem to $N$ and $T_{1}$, we obtain

$$
\begin{aligned}
& \mathbf{E}_{x}\left[e^{-\alpha X_{T_{1}}-\theta T_{1}} \mathbf{1}_{\left\{X_{T_{1}}<c\right\}}\right]+\mathbf{E}_{x}\left[e^{-\alpha c-\theta T_{1}} \mathbf{1}_{\left\{X_{T_{1}} \geq c\right\}}\right]=e^{-\alpha x} \mathbf{1}_{\{x<c\}}+e^{-\alpha c} \mathbf{1}_{\{x \geq c\}}-\frac{\alpha \mu_{1}+\theta}{\lambda} \\
& \mathbf{E}_{x}\left[e^{-\alpha X_{T_{1}}-\theta T_{1}} \mathbf{1}_{\left\{X_{T_{1}}<c\right\}}\right]-\frac{\theta e^{-\alpha c}}{\lambda} \mathbf{E}_{x}\left[e^{-\theta T_{1}} \mathbf{1}_{\left\{X_{T_{1}} \geq c\right\}}\right]+\frac{\alpha^{2}}{2} \frac{\lambda+\theta}{\lambda} \mathbf{E}_{x}\left[e^{-\theta T_{1}} \int_{0}^{T_{1}} e^{-\alpha X_{s}} \mathbf{1}_{\left\{X_{s} \leq c\right\}} d s\right]
\end{aligned}
$$

Note that we use two facts in the last equality. One is that $T_{1}$ is an exponential random variable which is independent of process $X$ and the other is $\mathbf{E}_{x}\left[e^{-\alpha X_{T_{1}}-\theta T_{1}} \mathbf{1}_{\left\{X_{T_{1}}=c\right\}}\right]=0$ and $\mathbf{E}_{x}\left[e^{-\theta T_{1}} \mathbf{1}_{\left\{X_{T_{1}}=c\right\}}\right]=0$. In addition, because of the definition of the function $g(\theta ; x)$, i.e., (3.11), we have

$$
g(\theta ; x)=\mathbf{E}_{x}\left[e^{-\theta T_{1}} \mathbf{1}_{\left\{X_{T_{1}} \geq c\right\}}\right]
$$

Moreover,

$$
\begin{aligned}
\mathbf{E}_{x}\left[e^{-\theta T_{1}} \int_{0}^{T_{1}} e^{-\alpha X_{s}} \mathbf{1}_{\left\{X_{s} \leq c\right\}} d s\right] & =\mathbf{E}_{x}\left[\int_{0}^{T_{1}} \int_{0}^{t} e^{-\alpha X_{s}} \mathbf{1}_{\left\{X_{s} \leq c\right\}} d s d e^{-\theta t}+\int_{0}^{T_{1}} e^{-\theta t} e^{-\alpha X_{t}} \mathbf{1}_{\left\{X_{t} \leq c\right\}} d t\right] \\
& =\mathbf{E}_{x}\left[-\int_{0}^{T_{1}} \theta e^{-\theta t} \int_{0}^{t} e^{-\alpha X_{s}} \mathbf{1}_{\left\{X_{s} \leq c\right\}} d s d t\right]+\frac{1}{\lambda} \Phi_{1}(\alpha, \theta ; x) \\
& =-\frac{\theta}{\lambda} \mathbf{E}_{x}\left[e^{-\theta T_{1}} \int_{0}^{T_{1}} e^{-\alpha X_{s}} \mathbf{1}_{\left\{X_{s} \leq c\right\}} d s\right]+\frac{1}{\lambda} \Phi_{1}(\alpha, \theta ; x) .
\end{aligned}
$$

Hence we have

$$
\mathbf{E}_{x}\left[e^{-\theta T_{1}} \int_{0}^{T_{1}} e^{-\alpha X_{s}} \mathbf{1}_{\left\{X_{s} \leq c\right\}} d s\right]=\frac{1}{\lambda+\theta} \Phi_{1}(\alpha, \theta ; x) .
$$

Combining (3.14) and (3.15), we arrive at (3.9). Finally we can derive (3.10) from (3.9) and (3.13). 
Remark 3.1. If there is no broken phenomenon in drift term, the equation (3.12) will be same as the equation (3.17) in Perry et al. (2004). Because of the broken phenomenon, the computations used in Perry et al. (2004) cannot be applied to this case. We need to construct a function by using timedependent tanaka formula to deal with the problem.

Proposition 3.2. For $0<x<b$, we can yield that

$$
\begin{aligned}
& \Phi_{3}(\alpha, \theta ; x)=\Phi_{1}(\alpha, \theta ; 0) \omega_{1}(\lambda+\theta ; b, x)+\Phi_{1}(\alpha, \theta ; b) \omega_{2}(\lambda+\theta ; b, x), \\
& \Phi_{4}(\alpha, \theta ; x)=\Phi_{2}(\alpha, \theta ; 0) \omega_{1}(\lambda+\theta ; b, x)+\Phi_{2}(\alpha, \theta ; b) \omega_{2}(\lambda+\theta ; b, x) .
\end{aligned}
$$

where $\omega_{1}(\lambda+\theta ; b, x)$ and $\omega_{2}(\lambda+\theta ; b, x)$ are given in (3.5) and (3.6) respectively, and $\Phi_{1}(\alpha, \theta ; 0), \Phi_{2}(\alpha, \theta ; 0)$ are given in Proposition 3.1.

Proof. Firstly, we divide $\Phi_{1}(\alpha, \theta ; x)$ into two parts.

$$
\Phi_{3}(\alpha, \theta ; x)=\mathbf{E}_{x}\left[e^{-\alpha X_{T_{1}}-\theta T_{1}} \mathbf{1}_{\left\{X_{\left.T_{1}<c\right\}}\right\}} \mathbf{1}_{\left\{\tau<T_{1}, X_{\tau}=0\right\}}\right]+\mathbf{E}_{x}\left[e^{-\alpha X_{T_{1}}-\theta T_{1}} \mathbf{1}_{\left\{X_{\left.T_{1}<c\right\}} \mathbf{1}_{\left\{\tau<T_{1}\right.}, X_{\tau}=b\right\}}\right]
$$

By using the strong Markov property of the process $X$, the memoryless property of the exponential distribution and the two equalities $T_{1}=\tau+T_{1} \circ \zeta_{\tau}$ and $X_{T_{1}}=X_{T_{1} \circ \zeta_{\tau}}$ on the event $\left\{\omega: \tau(\omega)<T_{1}(\omega)\right\}$, where $\zeta$. is the shift operator, we have that

$$
\begin{aligned}
\mathbf{E}_{x}\left[e^{-\alpha X_{T_{1}}-\theta T_{1}} \mathbf{1}_{\left\{X_{T_{1}<c}\right\}} \mathbf{1}_{\left\{\tau<T_{1}, X_{\tau}=0\right\}}\right] & =\mathbf{E}_{x}\left[\mathbf{E}_{x}\left(e^{-\alpha X_{T_{1}}-\theta T_{1}} \mathbf{1}_{\left\{X_{\left.T_{1}<c\right\}}\right.} \mathbf{1}_{\left\{\tau<T_{1}, X_{\tau}=0\right\}} \mid \mathcal{F}_{\tau}\right)\right] \\
& =\mathbf{E}_{x}\left[\mathbf{1}_{\left\{\tau<T_{1}, X_{\tau}=0\right\}} \mathbf{E}_{x}\left(e^{-\alpha X_{T_{1}}-\theta T_{1}} \mathbf{1}_{\left.\left\{X_{\left.T_{1}<c\right\}} \mid \mathcal{F}_{\tau}\right)\right]}\right]\right. \\
& =\mathbf{E}_{x}\left[e^{-\theta \tau} \mathbf{1}_{\left\{\tau<T_{1}, X_{\tau}=0\right\}} \mathbf{E}_{x}\left(e^{-\alpha X_{T_{1} \circ \zeta_{\tau}}-\theta T_{1} \circ \zeta_{\tau}} \mathbf{1}_{\left\{X_{T_{1} \circ \zeta_{\tau}<c}\right\}} \mid \mathcal{F}_{\tau}\right)\right] \\
& =\Phi_{1}(\alpha, \theta ; 0) \mathbf{E}_{x}\left[e^{-\theta \tau} \mathbf{1}_{\left\{\tau<T_{1}, X_{\tau}=0\right\}}\right] \\
& =\Phi_{1}(\alpha, \theta ; 0) \mathbf{E}_{x}\left[e^{-\theta\left(R_{0} \wedge R_{b}\right)} \mathbf{1}_{\left\{R_{0}<R_{b}\right\}} P\left(T_{1}>R_{0} \wedge R_{b} \mid X\right)\right] \\
& =\Phi_{1}(\alpha, \theta ; 0) \mathbf{E}_{x}\left[e^{-(\theta+\lambda) R_{0}} \mathbf{1}_{\left\{R_{0}<R_{b}\right\}}\right]
\end{aligned}
$$

Recall the definition of the function $\omega_{1}(\theta ; b, x)$ in $(3.1)$, we obtain

$$
\mathbf{E}_{x}\left[e^{-\alpha X_{T_{1}}-\theta T_{1}} \mathbf{1}_{\left\{X_{T_{1}<c}\right\}} \mathbf{1}_{\left\{\tau<T_{1}, X_{\tau}=0\right\}}\right]=\Phi_{1}(\alpha, \theta ; 0) \omega_{1}(\lambda+\theta ; b, x)
$$

Similarly,

$$
\mathbf{E}_{x}\left[e^{-\alpha X_{T_{1}}-\theta T_{1}} \mathbf{1}_{\left\{X_{T_{1}<c}\right\}} \mathbf{1}_{\left\{\tau<T_{1}, X_{\tau}=b\right\}}\right]=\Phi_{1}(\alpha, \theta ; b) \omega_{2}(\lambda+\theta ; b, x) .
$$

Combining (3.16) and (3.17), we arrive at the expression of $\Phi_{3}(\alpha, \theta ; x)$. Analogusly, we can obtain the expression of $\Phi_{4}(\alpha, \theta ; x)$. This completes the proof.

\subsection{Hitting problem of RBM with broken drift}

In this subsection, we shall study the first passage time $\tilde{\tau}$ defined in (1.5) and our main aim is to determine the joint Laplace transform:

$$
\tilde{\Psi}(\alpha, \theta ; x)=\mathbf{E}_{x}\left[e^{-\alpha \tilde{X} \tau-\theta \tilde{\tau}}\right]
$$


and similarly as the last section, we need to compute the following four Laplace transforms,

$$
\begin{aligned}
\tilde{\Phi}_{1}(\alpha, \theta ; x) & =\mathbf{E}_{x}\left[e^{-\alpha \tilde{X}_{T_{1}}-\theta T_{1}} \mathbf{1}_{\left\{X_{\left.T_{1}<c\right\}}\right\}}\right], \\
\tilde{\Phi}_{2}(\alpha, \theta ; x) & =\mathbf{E}_{x}\left[e^{-\alpha \tilde{X}_{T_{1}}-\theta T_{1}} \mathbf{1}_{\left\{X_{T_{1} \geq c}\right\}}\right], \\
\tilde{\Phi}_{3}(\alpha, \theta ; x) & =\mathbf{E}_{x}\left[e^{-\alpha \tilde{X}_{T_{1}}-\theta T_{1}} \mathbf{1}_{\left\{X_{\left.T_{1}<c\right\}}\right.} \mathbf{1}_{\left\{\tilde{\tau}<T_{1}\right\}}\right], \\
\tilde{\Phi}_{4}(\alpha, \theta ; x) & =\mathbf{E}_{x}\left[e^{-\alpha \tilde{X}_{T_{1}}-\theta T_{1}} \mathbf{1}_{\left\{X_{T_{1} \geq c}\right\}} \mathbf{1}_{\left\{\tilde{\tau}<T_{1}\right\}}\right] .
\end{aligned}
$$

Theorem 3.2. Let $0<x<b$. Then the joint LT of $\left(\tilde{X}_{\tilde{\tau}}, \tilde{\tau}\right)$ is given by

$$
\tilde{\Psi}(\alpha, \theta ; x)=e^{-\alpha b} \frac{\tilde{\psi}_{\theta+\lambda}(x)}{\tilde{\psi}_{\theta+\lambda}(b)}+e^{-\alpha b} \mathbf{E}_{x}\left[e^{-\theta T_{1}-\alpha Y_{1}} \frac{\tilde{\psi}_{\theta}(x)}{\tilde{\psi}_{\theta}\left(b+Y_{1}\right)} \mathbf{1}_{\left\{\tilde{\tau} \geq T_{1}\right\}}\right],
$$

where the last term on the right side can be determined by the following Proposition 3.3 and Proposition (3.4) and $\tilde{\psi}_{\theta+\lambda}(x)$ is given in $(2.5)$.

Proposition 3.3. Let $x \in \mathbb{R}$ and $\alpha, \theta>0$. Then

$$
\begin{aligned}
& \tilde{\Phi}_{1}(\alpha, \theta ; x)=\frac{\lambda\left(e^{-\alpha x} \mathbf{1}_{\{x<c\}}+e^{-\alpha c} \mathbf{1}_{\{x \geq c\}}\right)-(\lambda+\theta) e^{-\alpha c} g_{0}(\theta ; x)-\lambda \alpha g_{1}(\theta ; x)}{\lambda+\theta+\alpha \mu_{1}-\frac{1}{2} \alpha^{2}}, \\
& \tilde{\Phi}_{2}(\alpha, \theta ; x)=\frac{\lambda\left(e^{-\alpha x} \mathbf{1}_{\{x \geq c\}}-e^{-\alpha c} \mathbf{1}_{\{x \geq c\}}\right)+(\lambda+\theta) e^{-\alpha c} g_{0}(\theta ; x)}{\lambda+\theta+\alpha \mu_{2}-\frac{1}{2} \alpha^{2}},
\end{aligned}
$$

where $g_{0}(\theta ; x)=\mathbf{E}_{x}\left[e^{-\theta T_{1}} \int_{c}^{\infty} \tilde{p}\left(T_{1} ; x, y\right) d y\right], g_{1}(\theta ; x)=\frac{1}{2 \lambda} \mathbf{E}_{x}\left[e^{-\theta T_{1}} \tilde{p}\left(T_{1} ; x, 0\right)\right]$ and $\tilde{p}(t ; x, y)$ is the transition density function for the RBM with broken drift (i.e. stochastic process $\tilde{X}$ ) that can be obtained in $(2.6)$.

Proof. By applying Itô formula to $e^{-\alpha \tilde{X}_{t}-\theta t}$, we have

$$
\tilde{M}_{t}=e^{-\alpha \tilde{X}_{t}-\theta t}-e^{-\alpha \tilde{X}_{0}}+\left(\theta-\frac{1}{2} \alpha^{2}\right) \int_{0}^{t} e^{-\alpha \tilde{X}_{s}-\theta s} d s+\alpha \int_{0}^{t} \mu\left(\tilde{X}_{s}\right) e^{-\alpha \tilde{X}_{s}-\theta s} d s+\alpha \int_{0}^{t} e^{-\alpha \tilde{X}_{s}-\theta s} d L_{s}
$$

is a martingale. Then, applying the property of the local time $L_{t}$ in (1.3), we obtain

$$
\begin{aligned}
& \tilde{\Phi}_{1}(\alpha, \theta ; x)+\tilde{\Phi}_{2}(\alpha, \theta ; x)-e^{-\alpha x}+\left(\theta-\frac{1}{2} \alpha^{2}\right) \mathbf{E}_{x}\left[\int_{0}^{T_{1}} e^{-\alpha \tilde{X}_{s}-\theta s} d s\right] \\
& +\alpha \mathbf{E}_{x}\left[\int_{0}^{T_{1}} \mu\left(\tilde{X}_{s}\right) e^{-\alpha \tilde{X}_{s}-\theta s} d s\right]+\alpha \mathbf{E}_{x}\left[\int_{0}^{T_{1}} e^{-\theta s} d L_{s}\right]=0 .
\end{aligned}
$$

Similarly as the proof of the Proposition 3.1, we yield

$$
\frac{\lambda+\theta+\alpha \mu_{1}-\frac{1}{2} \alpha^{2}}{\lambda} \tilde{\Phi}_{1}(\alpha, \theta ; x)=e^{-\alpha x}-\alpha g_{1}(\theta ; x)-\frac{\lambda+\theta+\alpha \mu_{2}-\frac{1}{2} \alpha^{2}}{\lambda} \tilde{\Phi}_{2}(\alpha, \theta ; x),
$$

where the function $g_{1}(\theta ; x)$ is defined by

$$
g_{1}(\theta ; x)=\mathbf{E}_{x}\left[\int_{0}^{T_{1}} e^{-\theta s} d L_{s}\right]
$$

According to the equation (3.9) of Bo et al. (2011), the function $g_{1}(\theta, x)$ should be formulated by

$$
g_{1}(\theta ; x)=\frac{1}{2 \lambda} \mathbf{E}_{x}\left[e^{-\theta T_{1}} \tilde{p}\left(T_{1} ; x, 0\right)\right]
$$


Since $h(t, x)=e^{-\alpha x-\theta t} \mathbf{1}_{\{x<c\}}+e^{-\alpha c-\theta t} \mathbf{1}_{\{x \geq c\}}$ and $h(t, x)$ satisfies the conditions in Lemma 3.1, as a consequence, we get that

$$
\begin{aligned}
\tilde{N}_{t} & =e^{-\alpha \tilde{X}_{t}-\theta t} \mathbf{1}_{\left\{\tilde{X}_{t}<c\right\}}+e^{-\alpha c-\theta t} \mathbf{1}_{\left\{\tilde{X}_{t} \geq c\right\}}-e^{-\alpha x} \mathbf{1}_{\{x<c\}}-e^{-\alpha c} \mathbf{1}_{\{x \geq c\}}+\left(\alpha \mu_{1}+\theta\right) \\
& \int_{0}^{t} e^{-\alpha \tilde{X}_{s}-\theta s} \mathbf{1}_{\left\{\tilde{X}_{s} \leq c\right\}} d s+\theta \int_{0}^{t} e^{-\alpha c-\theta s} \mathbf{1}_{\left\{\tilde{X}_{s}>c\right\}} d s-\alpha^{2} \int_{\mathbb{R}} L^{\tilde{X}}(t, y) e^{-\alpha y-\theta t} \mathbf{1}_{\{y \leq c\}} d y \\
& -\alpha^{2} \theta \int_{\mathbb{R}} \int_{0}^{t} L^{\tilde{X}}(s, y) e^{-\alpha y-\theta s} \mathbf{1}_{\{y \leq c\}} d s d y+\alpha \int_{0}^{t} e^{-\alpha \tilde{X}_{s}-\theta s} \mathbf{1}_{\left\{\tilde{X}_{s} \leq c\right\}} d L_{s},
\end{aligned}
$$

is a martingale. It is similar to the proof of the Proposition 3.1 to obtain

$$
\tilde{\Phi}_{1}(\alpha, \theta ; x)=\frac{\lambda\left(e^{-\alpha x} \mathbf{1}_{\{x<c\}}+e^{-\alpha c} \mathbf{1}_{\{x \geq c\}}\right)-(\lambda+\theta) e^{-\alpha c} g_{0}(\theta ; x)-\lambda \alpha g_{1}(\theta ; x)}{\lambda+\theta+\alpha \mu_{1}-\frac{1}{2} \alpha^{2}} .
$$

Combining (3.20) and (3.21), we arrive at (3.19).

Proposition 3.4. For $0<x<b$, we have that

$$
\begin{aligned}
& \tilde{\Phi}_{3}(\alpha, \theta ; x)=\tilde{\Phi}_{1}(\alpha, \theta ; b) \frac{\tilde{\psi}_{\theta+\lambda}(x)}{\tilde{\psi}_{\theta+\lambda}(b)}, \\
& \tilde{\Phi}_{4}(\alpha, \theta ; x)=\tilde{\Phi}_{2}(\alpha, \theta ; b) \frac{\tilde{\psi}_{\theta+\lambda}(x)}{\tilde{\psi}_{\theta+\lambda}(b)},
\end{aligned}
$$

where $\tilde{\psi}_{\theta+\lambda}(x)$ is given in $(2.5)$, and $\tilde{\Phi}_{0}(\alpha, \theta ; 0)$ is given in Proposition 3.3.

Proof. The proof is similar to that of Proposition 3.2.

\section{Acknowledgement}

The authors are indebted to Professor Yongjin Wang for introducing them the topic of this research and many suggestions. This work is supported by the National Natural science Foundation of China (No. 11631004, 71532001).

\section{References}

Ata, B., Harrison, J. M., \& Shepp, L. A. (2005). Drift rate control of a Brownian processing system. Ann. Appl. Probab., 15, 1145-1160. URL: https://doi.org/10.1214/105051604000000855. doi:10.1214/105051604000000855.

Bo, L., Wang, Y., \& Yang, X. (2011). First passage times of (reflected) OrnsteinUhlenbeck processes over random jump boundaries. J. Appl. Probab., 48, 723-732. URL: https ://doi .org/10.1017/s0021900200008275. doi:10.1017/s0021900200008275.

Borodin, A. N., \& Salminen, P. (2002). Handbook of Brownian motion-facts and formulae. Probability and its Applications (2nd ed.). Birkhäuser Verlag, Basel. URL: https : //doi .org/10.1007/978-3-0348-8163-0. doi:10.1007/978-3-0348-8163-0. 
Che, X., \& Dassios, A. (2013). Stochastic boundary crossing probabilities for the Brownian motion. J. Appl. Probab., 50, 419-429. URL: https://doi.org/10.1239/jap/1371648950. doi:10.1239/jap/1371648950.

Elworthy, K. D., Truman, A., \& Zhao, H. (2007). Generalized Itô formulae and space-time LebesgueStieltjes integrals of local times. In Séminaire de Probabilités XL (pp. 117-136). Springer, Berlin volume 1899 of Lecture Notes in Math.. URL: https://doi.org/10.1007/978-3-540-71189-6_5. doi:10.1007/978-3-540-71189-6_5.

Harrison, J. M. (1990). Brownian motion and stochastic flow systems. Robert E. Krieger Publishing Co., Inc., Malabar, FL. Reprint of the 1985 original.

Jang, B.-G., \& Shim, G. (2008). A reflected diffusion process in a regime-switching environment. Oper. Res. Lett., 36, 177-183. URL: https://doi.org/10.1016/j.orl.2007.05.014. doi:10.1016/j.orl.2007.05.014.

Keilson, J., \& Wellner, J. A. (1978). Oscillating Brownian motion. J. Appl. Probability, 15, 300-310. URL: https ://doi .org/10.2307/3213403. doi:10.2307/3213403.

Perry, D., Stadje, W., \& Zacks, S. (2004). The first rendezvous time of Brownian motion and compound Poisson-type processes. J. Appl. Probab., 41, 1059-1070. URL: https://doi.org/10.1239/jap/1101840551. doi:10.1239/jap/1101840551.

Pilipenko, A. (2014). An introduction to stochastic differential equations with reflection. Universitätsverlag Potsdam.

Pötzelberger, K., \& Wang, L. (2001). Boundary crossing probability for Brownian motion. J. Appl. Probab., 38, 152-164. URL: https://doi.org/10.1017/s002190020001857x. doi:10.1017/s002190020001857x.

Revuz, D., \& Yor, M. (1999). Continuous martingales and Brownian motion volume 293 of Grundlehren der Mathematischen Wissenschaften [Fundamental Principles of Mathematical Sciences]. (3rd ed.). Springer-Verlag, Berlin. URL: https://doi.org/10.1007/978-3-662-06400-9. doi:10.1007/978-3-662-06400-9.

Su, F., \& Chan, K.-S. (2015). Quasi-likelihood estimation of a threshold diffusion process. J. Econometrics, 189, 473-484. URL: https://doi.org/10.1016/j.jeconom.2015.03.038. doi:10.1016/j.jeconom.2015.03.038.

Tong, H. (1983). Threshold models in nonlinear time series analysis volume 21 of Lecture Notes in Statistics. Springer-Verlag, New York. URL: https://doi.org/10.1007/978-1-4684-7888-4. doi:10.1007/978-1-4684-7888-4.

Wang, L., \& Pötzelberger, K. (1997). Boundary crossing probability for Brownian motion and general boundaries. J. Appl. Probab., 34, 54-65. URL: https://doi.org/10.2307/3215174. doi:10 . 2307/3215174. 
Yu, T.-H., Tsai, H., \& Rachinger, H. (2020). Approximate maximum likelihood estimation of a threshold diffusion process. Comput. Statist. Data Anal., 142, 106823, 14. URL: https://doi.org/10.1016/j.csda.2019.106823. doi:10.1016/j.csda.2019.106823. 